08

\title{
Особенности согласования нижнего электрода с высокочастотным генератором смещения при реактивно-ионном травлении массивных подложек
}

\author{
(С) С.Д. Полетаев, ${ }^{1,2}$ А.И. Любимов ${ }^{3}$ \\ ${ }^{1}$ Институт систем обработки изображений - филлиал Федерального научно-исследовательского центра \\ „Кристаллография и фоотоника“ РАН, \\ 443001 Самара, Россия \\ ${ }^{2}$ Самарский национальный исследовательский университет им. акад. С.П. Королева, \\ 443086 Самара, Россия \\ ${ }^{3}$ Научно-производственное объединение „Государственный институт прикладной оптики“, \\ 420075 Казань, Россия \\ e-mail: sergpolet@gmail.com
}

Поступило в Редакцию 18 сентября 2020 г.

В окончательной редакции 5 ноября 2020 г.

Принято к публикации 18 ноября 2020 г.

\begin{abstract}
Представлены теоретические и экспериментальные результаты по реактивно-ионному травлению массивных подложек во фреоне-14 с высокочастотным (ВЧ) смещением на нижнем электроде. Предложена гипотеза, согласно которой крупногабаритная подложка нарушает согласование нижнего электрода с ВЧ генератором за счет внесения дополнительной реактивной составляющей в импеданс нижнего электрода. Проведено численное моделирование реактивно-ионного травления с подложками различных габаритов в среде $\mathrm{CF}_{4}$. Результаты моделирования показали значительный рост реактивной составляющей ВЧ мощности на нижнем электроде, если диаметр подложки превышает 50\% диаметра нижнего электрода, что согласуется с предложенной гипотезой. Экспериментально показано, что при травлении массивных подложек нарушается согласование нижнего электрода с ВЧ генератором. Разработана специальная конструкция подложкодержателя для массивных подложек. Показано, что такой подложкодержатель существенно улучшает согласование ВЧ генератора с нижним электродом, особенно при добавлении в плазмообразующую смесь аргона в количестве $0.3-0.91 / \mathrm{h}$.
\end{abstract}

Ключевые слова: дифракционный микрорельеф, реактивно-ионное травление, метод конечных элементов, импеданс, подложкодержатель, высокочастотный индукционный разряд, нижний электрод, реактивная мощность.

DOI: $10.21883 / J T F .2021 .04 .50630 .271-20$

\section{Введение}

Для фокусировки ИК лазерного излучения высокой мощности в составе оптических схем применяются широкоапертурные дифракционные решетки, изготовленные на массивных пластинах [1,2]. Микрорельеф на таких пластинах обычно формируется либо механическим способом [3], либо путем химического травления [4]. Однако для эффективной работы с лазерными пучками видимого диапазона необходимы элементы с частотой штрихов свыше $1000 \mathrm{~mm}^{-1}$. Для формирования микрорельефа таких оптических элементов целесообразно применение метода реактивно-ионного травления (РИТ), основанном на ВЧ-индукционном (ВЧИ) разряде $[5,6]$. Однако в известных работах отсутствуют данные о влиянии массогабаритных характеристик подложек на процесс их травления в установках РИТ $[7,8]$. Важность данной связи для технологии плазменного травления связана с тем, что в установках РИТ для достижения высокой скорости и анизотропии травления дополнительно к ВЧИ разряду создается физический (ионный) компонент распыления. Для этого через согласующее устройство (СУ) на нижний электрод с установленной подложкой подается дополнительное напряжение от высокочастотного генератора (ГВЧ). Согласно техническому паспорту на установку РИТ Caroline PE15, коэффициент отражения по мощности на нижнем электроде при этом должен быть не более 25\%. Это требование выполняется при травлении стандартных кремниевых и других подложек, имеющих небольшие габариты и толщину до $5 \mathrm{~mm}$.

В настоящей работе исследуется влияние крупногабаритных подложек круглой формы с подложкодержателем на согласование нижнего электрода с ГВЧ в установках РИТ с привлечением численного эксперимента в программной среде Comsol multiphysics.

\section{1. Постановка задачи для моделирования}

Ключевым фактором, влияющим на согласование ГВЧ с нижним электродом, является импеданс нагрузки. Для 
его количественной и качественной оценки необходимо знать характер проводимости плазмы.

При относительно низких давлениях, когда частота возбуждения плазмы $\omega$ значительно превышает частоту столкновения электронов, диэлектрическую проницаемость можно определить по формуле [9]:

$$
\varepsilon \approx 1-\omega_{p}^{2} / \omega^{2}
$$

где $\omega_{p}-$ плазменная частота для электронного газа, определяемая по формуле:

$$
\omega_{p}=5.64 \cdot 10^{4} \sqrt{n_{e}},
$$

где $n_{e}-$ плотность свободных электронов.

В плазме ВЧ разряда плотность свободных электронов, как правило, превышает $10^{8} \mathrm{~cm}^{-3}$. Согласно формуле (2), при данной минимальной плотности плазменная частота $\omega_{p}=5.64 \cdot 10^{8} \mathrm{~s}^{-1}$, а диэлектрическая проницаемость $\varepsilon-$ большое отрицательное число, т.е. плазма является проводником. При этом условии в отсутствии подложки плазма является комплексной нагрузкой для ГВЧ, включающей активную и реактивную (преимущественно емкостную) составляющие. Подложка, превышающая по габаритам 50\% диаметра нижнего электрода, предположительно существенно меняет характер нагрузки, внося в общий импеданс дополнительный реактивный (емкостный) компонент. Этот компонент может быть обусловлен избыточным накоплением заряженных частиц на поверхностях подложки, что приводит к рассогласованию нижнего электрода с ГВЧ. Представляет практический интерес проверка данной гипотезы путем моделирования системы плазменного травления массивных подложек с ВЧИ разрядом и ВЧ смещением на нижнем электроде.

\section{2. Моделирование и результаты}

Для проверки выдвинутой гипотезы было проведено моделирование процесса РИТ массивной подложки с подачей ВЧ напряжения на нижний электрод в программной среде COMSOL Multiphysics v.5.2 с применением модулей Inductivle coupled plasma и AC/DC Electric current. Алгоритм моделирования основан на совместном решении уравнения индукционного тока и уравнения сохранения тока методом конечных элементов [10].

Порядок постановки и решения задачи в данной программной среде следующий [11]:

- задание геометрических параметров моделируемой системы;

- задание физических свойств материалов, необходимых для работы данного модуля;

- задание граничных условий;

- задание констант и выражений, действующих в различных областях подложки и за ее пределами;

- построение сетки;

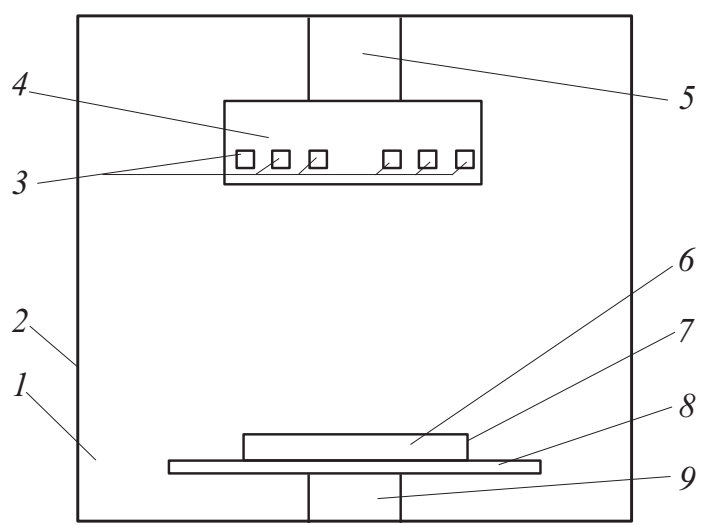

Рис. 1. Моделируемая система: 1 - камера, $2-$ стенка камеры, 3 - индуктор, 4 - изолятор $1,5-$ изолятор 2 , 6 - подложка, 7 - подложкодержатель, 8 - нижний электрод, 9 - изолятор.

- выбор и запуск решающего модуля;

- визуализация результатов.

Особенность моделирования заключалась в необходимости симуляции ВЧИ разряда в камере установки с ВЧ смещением, подаваемым на нижний электрод, что обусловило применение одновременно двух вышеназванных физических модуля.

На рис. 1 приведен вид моделируемой системы, представляющей собой вакуумную камеру с расположенными внутри индуктором, изоляторами, нижним электродом и подложкой. Каждый из конструктивных элементов в Comsol multiphsics является доменом с заданными свойствами. Моделирование проводилось для следующих вариантов:

1. Без подложки;

2. Подложки круглой формы толщиной 5, 10 и $15 \mathrm{~mm}$, когда подложкодержателем является сам нижний электрод;

3. Подложка толщиной $15 \mathrm{~mm}$, плотно установленная в подложкодержатель из алюминия, так, что с плазмой контактирует только верхняя сторона. Покрытие подложки с торцов токопроводящим материалом теоретически должно снижать реактивную составляющую импеданса, поскольку в этом случае снижается способность подложки накапливать заряженные частицы.

Моделирование ограничивалось двумерной моделью (2D-symmetry), поскольку конструкция вакуумной камеры симметрична. Размеры основных конструктивных элементов составили: диаметр камеры $300 \mathrm{~mm}$, диаметр нижнего электрода $200 \mathrm{~mm}$, индуктор с изолятором $140 \times 50 \mathrm{~mm}$. Подложка устанавливалась в подложкодержатель вышеописанной конструкции и имела размеры $120 \times 15 \mathrm{~mm}$. С целью достижения стабильного решения густота сетки задавалась таким образом, чтобы между любыми двумя границами было не меньше десяти конечных элементов.

В табл. 1-3 приводятся все заложенные в модель свойства доменов, глобальные параметры, электриче- 
Таблица 1. Домены и их принадлежность модулям

\begin{tabular}{c|c|c}
\hline Физический модуль & Индуктивно связанная плазма & AC/DC электрический ток \\
\hline \multirow{2}{*}{ Домены и материалы } & \multicolumn{2}{|c}{ Вакуумная камера - фреон 14} \\
\cline { 2 - 3 } & \multicolumn{2}{|c}{ Стенки камеры - сталь } \\
\cline { 2 - 3 } & Изоляторы $1,2-$ тефлон & Изолятор $3-$ тефлон \\
\cline { 2 - 3 } & Индуктор - медь & Нижний электрод, \\
\cline { 2 - 3 } & - & Подложодержатель - алюминий \\
\hline
\end{tabular}

Таблица 2. Актуальные характеристики материалов [12]

\begin{tabular}{c|c|c|c|c|c|c}
\hline Характеристика & Медь & Тефлон & Алюминий & Фреон 14 & $\begin{array}{c}\text { Сталь } \\
\text { Кварцевое } \\
\text { стекло }\end{array}$ \\
\hline $\begin{array}{c}\text { Проводимость } \\
10^{7}, \mathrm{~S} / \mathrm{m}\end{array}$ & 6 & - & 3.5 & - & 1.0 & - \\
\hline $\begin{array}{c}\text { Диэлектрическая } \\
\text { проницаемость }\end{array}$ & - & 2.1 & - & 1.0 & - & 4.3 \\
\hline $\begin{array}{c}\text { Магнитная } \\
\text { проницаемость }\end{array}$ & 1.0 & 1.0 & 1.0 & 1.0 & 2200 & 1.0
\end{tabular}

Таблица 3. Параметры доменов и границ

\begin{tabular}{|c|c|c|}
\hline Домены и границы & Параметр & Значение \\
\hline Индуктор & $\begin{array}{l}\text { Мощность индуктора } \\
\text { (частота } 13.56 \mathrm{MHz}), \mathrm{W}\end{array}$ & 800 \\
\hline $\begin{array}{c}\text { Изоляторы } 1,2, \text { индуктор, } \\
\text { камера }\end{array}$ & Температура, K & 300 \\
\hline \multirow{2}{*}{ Камера } & Давление, $\mathrm{Pa}$ & 0.2 \\
\hline & $\begin{array}{c}\text { Подвижность электронов, } \\
\mathrm{m}^{2} /(\mathrm{V} \cdot \mathrm{s})\end{array}$ & 30 \\
\hline $\begin{array}{c}\text { Границы подложки и } \\
\text { нижнего электрода }\end{array}$ & $\begin{array}{c}\text { Напряжение на нижнем } \\
\text { электроде } \\
\text { (частота } 13.56 \mathrm{MHz}), \mathrm{V}\end{array}$ & $200 \cdot \sin (13560000 \cdot t)$ \\
\hline \multirow{2}{*}{ Стенки камеры } & Общий, V & 0 \\
\hline & Коэффициент отражения & 0.2 \\
\hline
\end{tabular}

ские сигналы и граничные условия. Прочерком показаны величины, не участвующие в расчетах или участвующие в неявном виде. Моделирование проводилось при давлении, характерном для тлеющего разряда. Для симуляции ионной составляющей травления, обусловливающей ток через нижний электрод, на него подавалось переменное напряжение с частотой $13.56 \mathrm{MHz}$. Нулевым потенциалом для обоих модулей является корпус камеры.

На разрядной частоте $13.56 \mathrm{MHz}$ и $p<10$ Ра подвижность электронов составляет $\sim 50 \mathrm{~m}^{2} /(\mathrm{V} \cdot \mathrm{s})$ [9].
В условиях плазмы в среде фреона 14 возможно протекание реакций по следующим схемам [13]:

$$
\begin{gathered}
e+C F_{4} \rightarrow C F_{3}^{+}+F^{\bullet}+2 e, \\
e+C F_{4} \rightarrow C F_{3}^{\bullet}+F^{\bullet}+e, \\
e+C F_{4} \rightarrow C F_{3}^{\bullet}+F^{-}, \\
C F_{3}^{\bullet}+F^{\bullet} \rightarrow C F_{4},
\end{gathered}
$$




$$
\begin{gathered}
F^{\bullet}+F^{\bullet} \rightarrow F_{2}, \\
C F_{3}^{\bullet}+C F_{3}^{\bullet} \rightarrow C_{2} F_{6} .
\end{gathered}
$$

Из всех этих реакций в модели учитывались реакции (4)-(7), как энергетически наиболее вероятные.

Расчет велся решателем Frequency-Trancient в интервале $10^{-8}-10^{-3} \mathrm{~s}$ для 21 значений времени, распределенных равномерно. Данный решатель применяется для вычисления изменений температуры во времени вместе с распределением электромагнитного поля в частотной области. Временной интервал определялся, исходя из установления стационарного режима плазмы [14].

В модели не учитывалась временная зависимость температуры доменов, поскольку в практически значимых случаях она изменяется несущественно (в пределах $15 \%$ ) и не оказывает существенного влияния на результат решения.
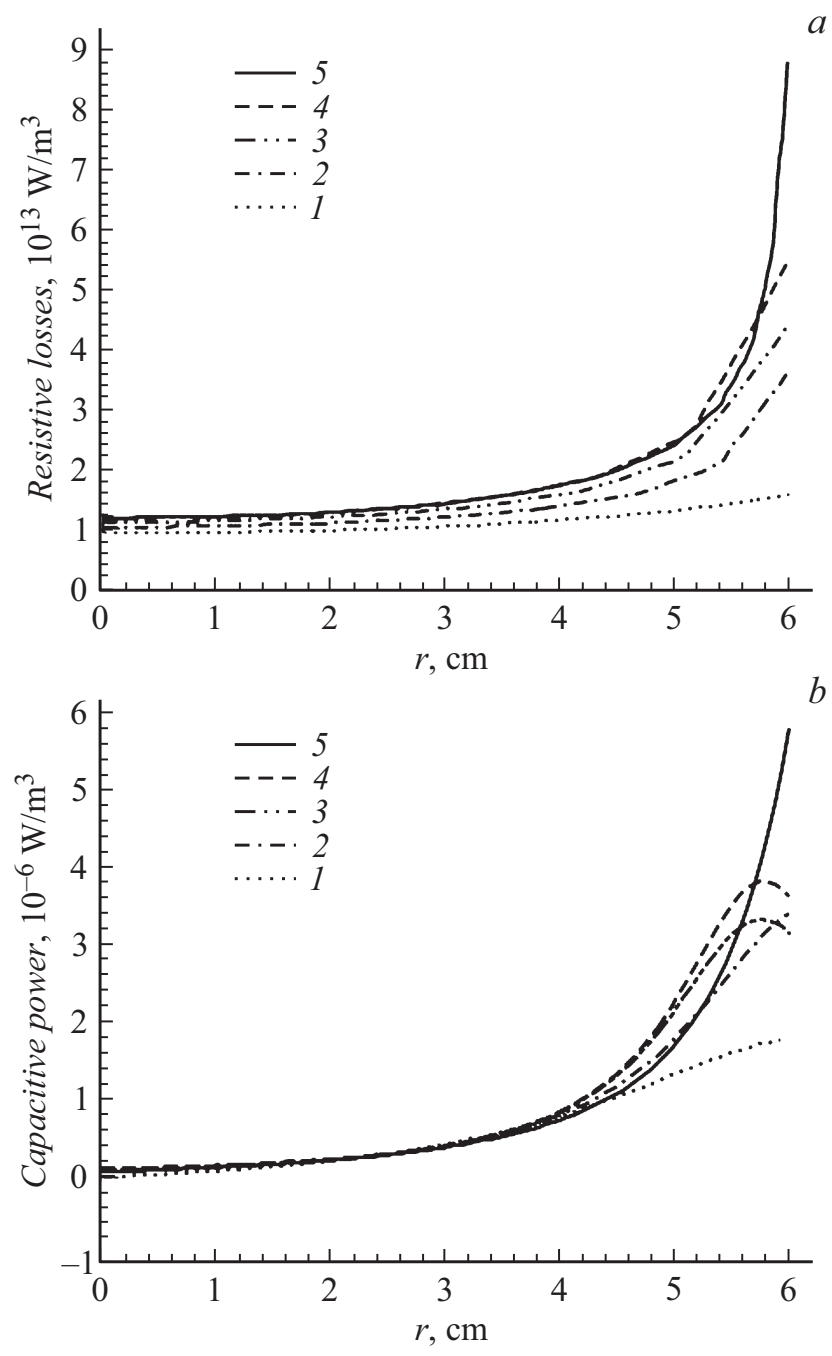

Рис. 2. Распределение резистивных потерь $(a)$ и удельной реактивной (емкостной) мощности $(b)$ по поверхности подложки вдоль радиуса: $1-$ без подложки, $2-$ с подложкой $h=5 \mathrm{~mm}$, $3-$ с подложкой $h=10 \mathrm{~mm}, 4-$ с подложкой $h=15 \mathrm{~mm}$, $5-$ с подложкой $h=15 \mathrm{~mm}$ и подложкодержателем; $t=1 \mathrm{~ms}$.
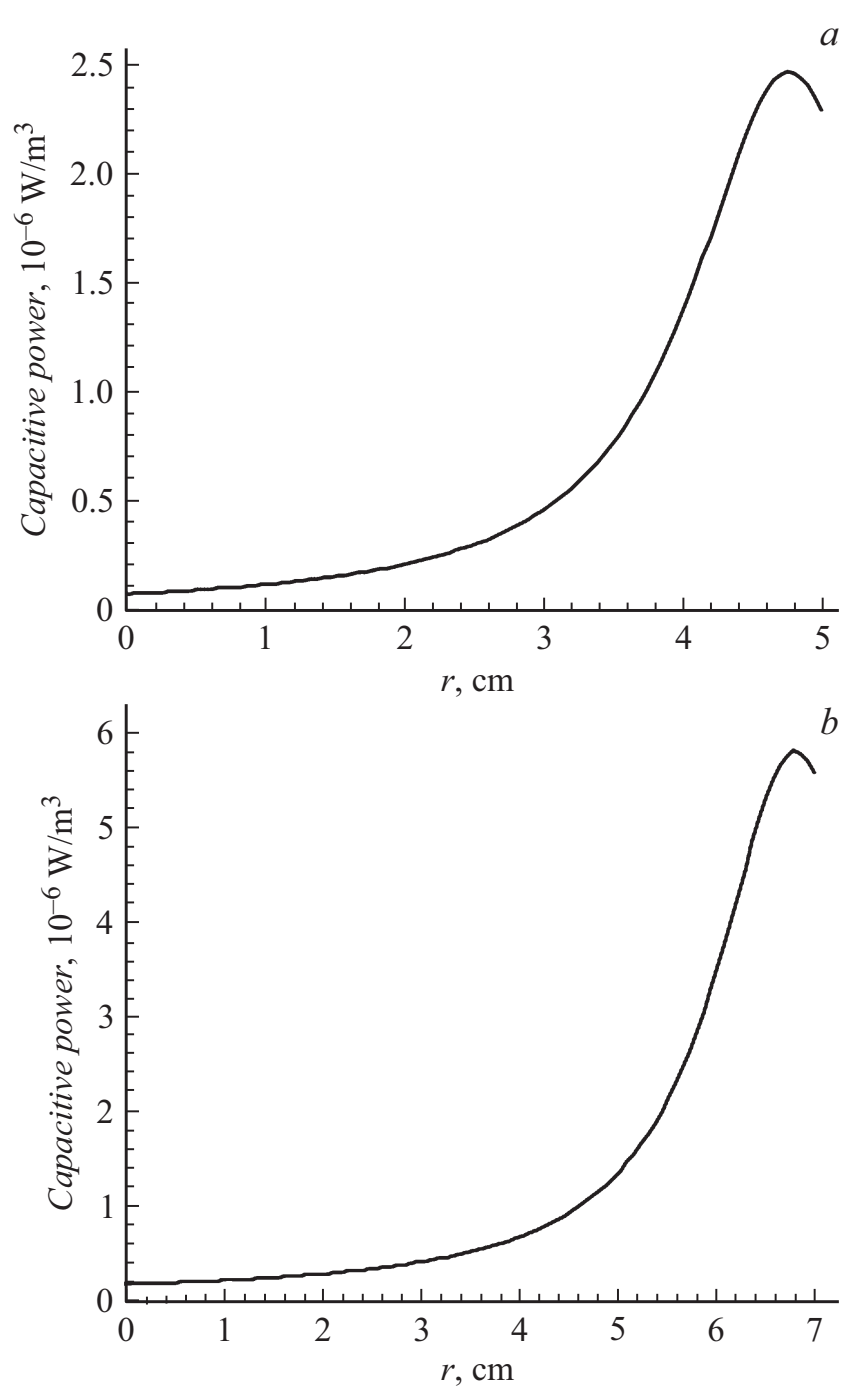

Рис. 3. Распределение удельной реактивной мощности по поверхности подложек размерами $100 \times 15(a)$ и $140 \times 15 \mathrm{~mm}(b) ; t=1 \mathrm{~ms}$.

На рис. 2 показано семейство распределений резистивных потерь и удельной реактивной (емкостной) мощности вдоль поверхности для подложек с различной толщиной. Из рис. 2, $a$ видно, что увеличение толщины подложки до $15 \mathrm{~mm}$ приводит к последовательному росту резистивных потерь. При $r=4 \mathrm{~cm}$ их увеличение составляет около $60 \%$. Однако графики резистивных потерь для подложки толщиной 15 mm с подложкодержателем и без него практически идентичны, т. е. по данному показателю подложкодержатель влияния не оказывает. Также видно, что до $r=4 \mathrm{~cm}$ резистивные потери почти не изменяются, а их заметный рост наблюдается только при $r>4 \mathrm{~cm}$.

Поведение удельной реактивной мощности заметно отличается от поведения резистивных потерь. До $r=3 \mathrm{~cm}$ реактивная мощность увеличивается приблизительно в 3 раза, а графики на этом участке выглядят идентично. И только при $r>3 \mathrm{~cm}$ начинается 

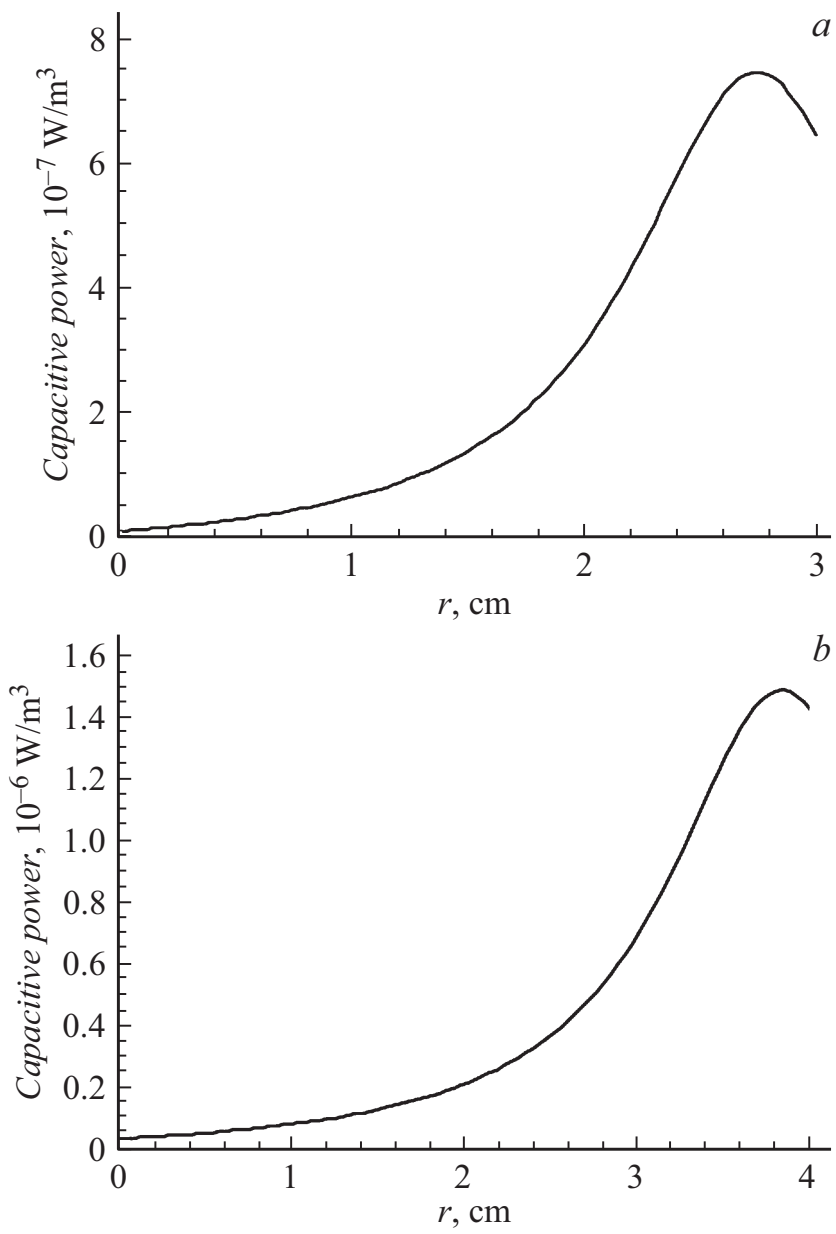

Рис. 4. Распределение удельной реактивной мощности по поверхности подложек размерами $60 \times 15(a)$ и $80 \times 15 \mathrm{~mm}(b)$; $t=1 \mathrm{~ms}$.

их расхождение. При $r=4 \mathrm{~cm}$ реактивная мощность увеличивается приблизительно на $30 \%$ с увеличением толщины подложки до $15 \mathrm{~mm}$. Важно отметить, что кривая 5, соответствующая подложке с подложкодержателем вплоть до $r=5.7 \mathrm{~cm}$, идет существенно ниже кривой, соответствующей подложке толщиной $15 \mathrm{~mm}$, что означает снижение эффекта вносимой мощности. Рост значений представленных выше характеристик с увеличением толщины подложки означает увеличение степени рассогласования ГВЧ с нижним электродом.

Еще одной особенностью распределения удельной реактивной мощности является резкий рост значений вблизи края подложки. Для объяснения причин этого было проведено моделирование с подложками размером $100 \times 15$ и $140 \times 15 \mathrm{~mm}$ без подложкодержателя. На рис. 3 показано распределение реактивной мощности для этих случаев. Из рисунка видно, что на краю значение минимально $\left(\sim 2.5 \mu \mathrm{W} / \mathrm{m}^{3}\right)$ для подложки диаметром $100 \mathrm{~mm}$ и максимально $\left(\sim 6.0 \mu \mathrm{W} / \mathrm{m}^{3}\right)$ для подложки диаметром $140 \mathrm{~mm}$. Для подложки диаметром $120 \mathrm{~mm}$ реактивная мощность принимает промежуточное зна- чение $\left(\sim 4.0 \mu \mathrm{W} / \mathrm{m}^{3}\right)$. Это означает, что быстрый рост реактивной мощности на краю подложки обусловлен исключительно вносимой удельной емкостью и не связан с другими факторами, в качестве наиболее вероятного из которых выступает коронный эффект на остром крае [15]. В последнем случае максимальное значение реактивной мощности не зависело бы от линейного размера подложки. В свою очередь, зависимость удельной емкости от линейного размера подложки, очевидно, вызвана конструктивными особенностями рабочей камеры. Объяснение резкого роста реактивной мощности на краю коронным эффектом возможно только для варианта с подложкодержателем, конструкция которого включает острые металлические края. В соответствии с этим из рис. $2, b$ видно, что, действительно, максимальное значение реактивной мощности на краю подложки соответствует варианту с подложкодержателем. Также видно, что увеличение диаметра подложки на $40 \%$ (от 100 до $140 \mathrm{~mm}$ ) приводит к резкому росту реактивной мощности (в 2.4 раза), что означает значительное ухудшение условий согласования.

На рис. 4 показаны графики распределения удельной реактивной мощности для подложек размером $60 \times 15$ и $80 \times 15 \mathrm{~mm}$. Видно, что уменьшение диаметра подложки приводит к последовательному снижению реактивной мощности на краю подложки от $\sim 2.5 \mu \mathrm{W} / \mathrm{m}^{3}$ для диаметра $100 \mathrm{~mm}$ до $\sim 0.7 \mu \mathrm{W} / \mathrm{m}^{3}$ для диаметра $60 \mathrm{~mm}$. При этом уменьшение диаметра от 80 до $60 \mathrm{~mm}$ приводит к двукратному снижению реактивной мощности. По всей видимости, диаметр подложки в пределах 60-80 mm является предельным, не влияющим на согласование нижнего электрода с ГВЧ.

На рис. 5 представлено распределение объемной плотности заряда химически активных частиц (ХАЧ) плазмы $(Q)$ вдоль радиуса до $60 \mathrm{~mm}$ для подложек

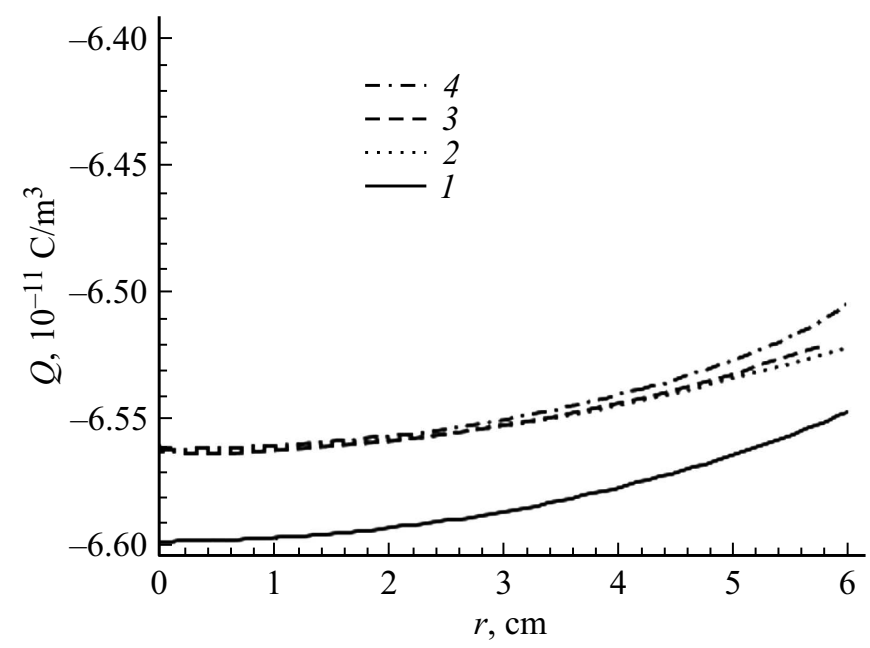

Рис. 5. Распределение плотности заряда ХАЧ плазмы: 1 - без подложки по поверхности нижнего электрода и на расстоянии $25 \mathrm{~mm}$ от него, 2 - по поверхности подложки $h=5 \mathrm{~mm}$, 3 - по поверхности подложки $h=15 \mathrm{~mm}, 4-$ по поверхности подложки $h=15 \mathrm{~mm}$ с подложкодержателем; $t=1 \mathrm{~ms}$. 
различной толщины. Видно, что независимо от толщины подложки по направлению к краю наблюдается несущественный (менее $1 \%, \Delta Q=0.04 \mathrm{C} / \mathrm{m}^{3}$ ) рост данного параметра, и даже внесение подложкодержателя в плазму не меняет характера распределения. Внесение подложки приводит также к снижению общей плотности заряда ХАЧ плазмы менее чем на $1 \%$, не зависящему от толщины. Кривые 3,4, соответствующие подложке толщиной $15 \mathrm{~mm}$ с подложкодержателем и без него, практически неотличимы друг от друга и лишь на самом краю наблюдается расхождение $\Delta Q=0.01 \mathrm{C} / \mathrm{m}^{3}$. Данные рис. 5 должны свидетельствовать о равномерности процесса травления независимо от толщины подложки и наличия подложкодержателя.

\section{3. Разработка конструкции подложкодержателя}

Вышеприведенные результаты моделирования показывают, что в случае, когда методом РИТ производится травление массивной подложки, диаметр которого превышает 50\% диаметра нижнего электрода, а толщина превышает $10 \mathrm{~mm}$, условия согласования нарушаются. При этом встроенные $L C$-элементы CУ не могут компенсировать возникающий при этом дисбаланс мощностей, поэтому необходим поиск других способов улучшения согласования массивных подложек, установленных на нижнем электроде, с ГВЧ. Одним из таких способов может быть применение держателей специальной конструкции, снижающей вносимую реактивную составляющую импеданса нагрузки.

В технологии плазменного осаждения и травления материалов часто применяются подложкодержатели с вакуумным прижимом [16]. Их конструкция включает в себя нижний электрод, в котором изготовлены проточки, соединенные с вакуумным насосом. Поверх электрода устанавливается резиновое кольцо, на котором размещается подложка. Резиновое кольцо уплотняет пространство между подложкой и электродом, фиксируя подложку. Такая конструкция за счет резинового уплотнителя имеет большую емкость электрода относительно корпуса установки, что ухудшает условия для согласования подложки и генератора.

Также распространен подложкодержатель в виде металлической пластины [17]. В пластину монтируется подложка и устанавливается на нижний электрод. В других вариантах исполнения нижний электрод сам

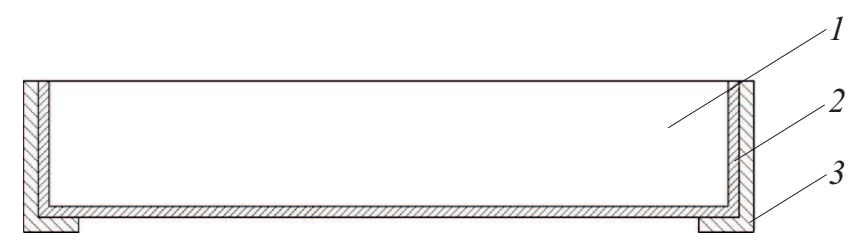

Pис. 6. Конструкция подложкодержателя: 1 - подложка, 2 - фольга, 3 - оправка. является подложкодержателем. Такая конструкция может иметь по краям дополнительные прижимы для лучшей фиксации подложек. Для улучшения теплоотвода на поверхность пластины наносится специальный теплопроводящий клей или вакуумная смазка. Такая конструкция проста, но, как показали вышеприведенные результаты моделирования, ее недостаток заключается в невозможности согласования массивных подложек с ВЧ генератором смещения.

С учетом плохих условий согласования простейшего подложкодержателя, на практике была реализована конструкция, показавшая в процессе моделирования улучшенные результаты (рис. 6). Подложка 1, предназначенная для травления, равномерно покрывается алюминиевой фольгой 2 таким образом, чтобы металлическая поверхность закрывала основание и торцы подложки. Затем подложка с фольгой помещается в алюминиевую оправку 3 со стороны основания подложки таким образом, чтобы оправка полностью закрывала подложку с торцов. Оправка изготавливается по форме подложки и имеет Г-образный профиль. Толщина оправки должна обеспечивать ее минимальную механическую прочность. Оправка также может устанавливаться сверху, однако в этом случае велика вероятность повреждения и отслоения фольги от основания подложки. В целом фольга и оправка могут быть из любого материала с высокой электропроводностью.

\section{4. Эксперимент}

Ниже описан эксперимент, показывающий влияние размера подложки на согласование ГВЧ с нижним электродом в установке РИТ Caroline PE15 без добавки аргона. Мощность на индукторе $400 \mathrm{~W}$. Подложка размером $120 \times 15 \mathrm{~mm}$ устанавливалась на нижний электрод, имеющий круглую форму диаметром $200 \mathrm{~mm}$. Расход газов составлял $1.01 / \mathrm{h}$. Согласование оценивалось по коэффициенту отражения падающей на электрод ВЧ мощности. Результаты, полученные с вариантом [17], представлены в первой строке табл. 4. Из данных следует, что ГВЧ в этом случае не согласован с нижним

Таблица 4. Коэффициенты отражения по мощности, полученные на нижнем электроде для установки реактивно-ионного травления Caroline PE15 с различными газами и подложкодержателями

\begin{tabular}{c|r|c|c|c}
\hline \multirow{2}{*}{ Подложкодержатель } & \multicolumn{4}{|c}{$P_{\text {refl }} / P_{\text {inc }}, \%$} \\
\cline { 2 - 5 } & $\mathrm{CHF}_{3}$ & $\mathrm{CF}_{4}$ & $\mathrm{SF}_{6}$ & $\mathrm{Ar}$ \\
\hline Простейший & 100 & 75 & 45 & 28 \\
\hline Исследуемый & 62 & 47 & 15 & 15
\end{tabular}

Примечание. Размер подложки $120 \times 15 \mathrm{~mm}$, диаметр нижнего электрода $200 \mathrm{~mm}$. 
Таблица 5. Влияние добавки аргона на согласование ГВЧ с нижним электродом и подложкодержателем в среде фреонов

\begin{tabular}{c|c|c}
\hline \multirow{2}{*}{$\begin{array}{c}\text { Рacход } \\
\text { Ar, } 1 / \mathrm{h}\end{array}$} & \multicolumn{2}{|c}{$P_{\text {refl }} / P_{\text {inc }}, \%$} \\
\cline { 2 - 3 } & $\mathrm{CHF}_{3}$ & $\mathrm{CF}_{4}$ \\
\hline 0 & 62 & 60 \\
0.3 & 52 & 46 \\
0.6 & 30 & 27 \\
0.9 & 21 & 18 \\
1.2 & 16 & 15 \\
1.5 & 15 & 14
\end{tabular}

Примечание. Расход фреонов $1.01 / \mathrm{h}$.

электродом (соотношение мощностей выше 25\%). Коэффициент отражения по мощности с подложкодержателем вышеописанной конструкции представлен во второй строке табл. 4. Видно, что с таким подложкодержателем согласование в плазме чистых газов значительно улучшается. Для $\mathrm{SF}_{6}$ коэффициент соответствует паспортному значению. Такое влияние подложкодержателя на согласование хорошо соответствует результатам моделирования. В последнем столбце для сравнения приведены данные для Ar. Видно, что даже без подложкодержателя с этим газом коэффициент отражения близок к $25 \%$. Это свойство аргона может быть использовано для дальнейшего улучшения согласования в плазме с фреонами, а его концентрация в общем случае определяется требованиями к коэффициенту отражения по мощности, режимом травления и материалом подложки.

В табл. 5 представлены данные по согласованию ГВЧ с нижним электродом в среде фреонов $\mathrm{CHF}_{3}$ и $\mathrm{CF}_{4}$ для массивной подложки с подложкодержателем и добавкой аргона. Видно, что даже небольшая добавка аргона с расходом $0.31 / \mathrm{h}$ существенно снижает коэффициент отражения. Оптимальное значение расхода аргона находится в пределах 0.6-0.91/h. Нужно отметить, что такой подход к согласованию не может быть применим в случаях, когда добавка аргона в указанных количествах существенным образом ухудшает характеристики травления, в первую очередь шероховатость поверхности и скорость травления подложки.

\section{Заключение}

В работе представлены теоретические и экспериментальные результаты по реактивно-ионному травлению массивных подложек во фреоне 14.

Экспериментально подтверждена гипотеза, согласно которой процесс РИТ подложек, диаметр которых превышает 50\% диаметра электрода, характеризуется значительной долей реактивной составляющей импеданса нижнего электрода, что приводит к его рассогласованию с ГВЧ смещения, некомпенсируемому согласующим устройством. Снижение данного эффекта возможно с применением подложкодержателя специальной конструкции. Такой подложкодержатель может быть применен для создания элементов дифракционной оптики на массивных подложках методом РИТ, работающих в схемах с лазерами высокой мощности.

\section{Благодарности}

Работа выполнена в рамках госзадания „Кристаллография и фотоника“ РАН/ (соглашение № 007-ГЗ/43363/26) и договора о НИР № 08/2017 (заказчик НПО „Государственный институт прикладной оптики“').

\section{Конфликт интересов}

Авторы заявляют, что у них нет конфликта интересов.

\section{Список литературы}

[1] N.L. Kazanskiy, G.V. Uspleniev, A.V. Volkov. Proceedings of SPIE, 4316, 193 (2000). DOI: 10.1117/12.407678

[2] P.A. Hilton, D. Lloyd, J.R. Tyrer. J. Laser. Appl., 28 (1), 012014-1 (2016). DOI: 10.2351/1.4938279

[3] С.Р. Абульханов, Н.Л. Казанский, Л.Л. Досколович, О.Ю. Казакова. СТИН, 9, 22 (2011).

[4] Z. Huang, N. Geyer, P. Werner, J. de Boor, U. Gösele. Adv. Mater., 23, 285 (2011). DOI: 10.1063/1.4817424

[5] Ю.П. Райзер, М.Н. Шнейдер, Н.А. Яценко. Высокочастотный емкостный разряд (Наука, М., 1995)

[6] Б.С. Данилин, В.Ю. Киреев. Применение низкотемпературной плазмы для травления и очистки материалов (Энергоатомиздат, М., 1987)

[7] V.M. Donnellya, A. Kornblit. J. Vac. Sci. Technol., 31 (5), 050825-1 (2013). DOI: 10.1116/1.4819316

[8] C.C. Welch, D.L. Olynick, Z. Liu, A. Holmberg, C. Peroz, A.P.G. Robinson, M.D. Henry, A. Scherer, T. Mollenhauer, V. Genova, K.T.Ng. Doris. Proc. of SPIE, 8700 (870002), 870002-1 (2012). DOI: 10.1117/12.2017609

[9] Ю.П. Райзер. Физика газового разряда (Наука, М., 1987)

[10] Р.В. Хемминг. Численные методы для научных работников и инженеров (Наука, М., 1972)

[11] В.И. Егоров. Применение ЭВМ для решения задач теплопроводности. Учебное пособие (СПб ГУ ИТМО, СПб, 2006)

[12] И.С. Григорьев, Е.3. Мейлихов (ред.). Физические величины: Справочник (Энергоатомиздат, М., 1991)

[13] Г.Ф. Ивановский, В.И. Петров. Ионно-плазменная обработка материалов (Радио и связь, М., 1986)

[14] Э.Х. Исакаев, О.А. Синкевич, В.Б. Мордынский, А.С. Тюфтяев, А.Г. Хачатурова. Физика и химия обработки материалов, 2, 25 (2011).

[15] Ф.П. Гросу, А.М. Болога, М.К. Болога, О.В. Моторин. Электронная обработка материалов, 51 (5), 45 (2015).

[16] А.Ю. Данилов, О.А. Королев, В.Я. Первушин, Д.Е. Синицын. Патент SU1061196A. H01L 21/68.

[17] E.R. Parker, B.E. Thibeault, M.F. Aimi, M.P. Rao, N.C. Mac Donald. J. Electrochem. Soc., 152 (10), 675 (2005). 